Canadian Studies in Population, Vol. 30(1), 2003, pp. 71-73

\title{
Introduction to Anatole Romaniuc's Contributions to Aboriginal Demography
}

\author{
Frank Trovato \\ Department of Sociology \\ The University of Alberta \\ Edmonton, Alberta
}

\begin{abstract}
Anatole Romaniuc's fascination with the demography of Canada's Aboriginal peoples has perhaps something to do with his work in Africa prior to coming to this country in 1964. He found affinities between African and Aboriginal demographics and felt that the knowledge he has acquired in Africa and at Princeton University while working on the African Demographic Project can to some extent be transferred to Canada's Aboriginal environment. Shortly after joining the University of Ottawa he, with a team of students, initiated and successfully completed in 1968 a socio-demographic survey covering six aboriginal villages in the James Bay region. This survey has the merit of having captured the demographic configuration of Aboriginal peoples just shortly before they set on the course of demographic transition. It still remains the most comprehensive, detailed socio-demographic account of Aboriginal demography in Canada. He recalls having received $\$ 2,000$ from Canada Council (the predecessor of the SSHRC) to carry out the survey---a small amount even when our nationally currency had still its worth. Yet, the return from this modest financial investment was exceptionally rich: half a dozen MA theses by the students who participated in the survey, a dozen articles and papers by Romaniuc, and a host of scholars have also used the James Bay Indians data in their own research.
\end{abstract}


His contribution to the demography of Aboriginal people is both substantive and theoretical. For instance, he has written several papers on the procreative behaviors of Aboriginal peoples, the most recent being "Dynamics of Aboriginal Population Growth Under the Conditions of Encounter of Civilizations" (reprinted in this issue). This is a most authoritative statement on how their demographic fortune has evolved over time, since the early contacts with Europeans to our days. According to Anatole Romaniuc, the demographic regime of the pre- and early contact era was that of a precarious demographic equilibrium. Unlike many others he did not try to put a figure on what may have been the size of Indian population prior to the coming of Europeans. $\mathrm{He}$ considered this to be an impossible task. Instead he tried to describe the demographic regime that most likely prevailed in those days by a careful consideration of the biotic and living conditions, the state of technology and political organization. Europeans brought with infectious diseases against which Aboriginals lacked immunity and which brought about the collapse of traditional demographic equilibrium and an almost three centuries of depopulation. The recovery started at the turn of the century, and a demographic boom has taken place in the postwar decades. Now the Aboriginals are well into the demographic transition, but they have still some way to go before reaching parity with demographics of Canadian population. Such is the broad configuration of the demographic evolution of the Aboriginal people, according to Anatole Romaniuc.

A central feature of Romaniuc's work is his concerted interest in the theoretical underpinning of demographic research. Thus, any theoretical framework designed to assist in exploring and explaining the Aboriginals' demographic behavior must take into account features specific to their historical evolution and their special sociopolitical status. A simple transfer of theoretical models based on the demographic transition historically experienced by European or by contemporary developing nations is bound to miss many unique features of Aboriginal demographic experience. Nor easily transferable are many of the concepts and models forged by sociological theories concerning minority groups in North America (e.g., minority group status insecurity and assimilation theses). Any conceptualization of an explanatory framework of demographic transition of Aboriginals must embody features of the unique status they hold historically within the broader configuration of Canadian society. Romaniuc's model of Aboriginal demography rests on two conceptual pillars: one is what may be called ethnocentrism, the other dependency. In combination, these two features have resulted in a duality of Aboriginal identity, 'man of two cultures', or an 'in-between' society'; these conditions have contributed to a substantial delay in the Aboriginal fertility transition.

Another area of Anatole Romaniuc's research that involves theoretical conceptualization is the rising cycle of fertility associated with early modernization which he observed among Native people and which preceded the onset of transition to modern low fertility. At the time, this finding stood at odds 
with the prevailing orthodoxy, whereby it was thought that modernization entails automatically a monotonic decline in fertility. The occurrence of a rising cycle of natural fertility during the early stage of modernization, according to Romaniuc, can be rationalized as follows: the actual childbearing performance of traditional societies is well below potential because of the presence of various biological and cultural inhibiting factors. When traditional societies enter the processes of modernization these childbearing inhibiting factors are removed or weakened. His research in Africa revealed that an increase in natural fertility was primarily caused by improvements in health, more specifically by a reduction in sterility attributed to venereal diseases and, to some extent, by the breakdown of customs of prolonged post-natal abstinence associated with nursing of infants. Data for Canadian Indians bring to light the impact on natural fertility of a massive, almost abrupt, shift from breast-feeding to bottle-feeding. He noticed that a high proportion of younger Canadian Indian women either did not breastfed their babies or did only for a short period. This resulted in an early resumption of ovulation and ultimately in higher conception rates. This shift in lactation practices occurred prior to the generalization of birth control practices among Canadian Indians and this resulted in a rise in their fertility rate. The birth rate jumped from about 40 to about 47 in the two post war decades.

Notwithstanding his many contributions to this important area of social demography, Anatole Romaniuc has not yet said the last word on this topic. His research continues. As this note is being written, he is co-authoring a book on Aboriginal demography. No doubt, we can expect further important insights in his work about this demographically important and unique population in the Canadian ethnic mosaic.

Frank Trovato 